\title{
Effect of Self-Esteem in the Relationship between Stress and Substance Abuse among Adolescents: A Mediation Outcome
}

\author{
Ikechukwu Uba, Siti Nor Yaacob, Mansor Abu Talib, Sakineh Mofrad, and Rohani Abdullah
}

\begin{abstract}
The present study assessed the mediating effect of self-esteem in the relationship between stress and substance abuse among adolescents. The participants of the study were 352 adolescents, $54.5 \%$ males and $45.5 \%$ females aged 13 to18 years, from selected secondary schools in Somolu, Lagos, Nigeria. Substance abuse was measured with the Drug Abuse Screening Test, while Stress was measured with the Perceived Stress Scale, and Self-esteem was measured with the Rosenberg Self-esteem scale. The study ascertained a negative and large correlation $(r=-.538, p<.01)$ between stress and self-esteem, similar results $(r=-.536, p<.01)$ was found between self-esteem and substance abuse and a positive and medium correlation $(r$ $=.360, p<.01$ ) was found between stress and substance abuse. Self-esteem partially mediated the relationship between stress and substance abuse. Recommendations of the study highlighted the need for greater enlightenment on the importance of self-esteem particularly among adolescents.
\end{abstract}

Index Terms-Adolescence, self-esteem, stress, substance abuse

\section{INTRODUCTION}

In recent years, there has been increasing concerns that substance abuse poses great danger to addicts, those around them and the society at large [1]. Research has proven that adolescent substance abuse heightens the risk of nicotine dependence, alcohol, marijuana and other illicit drug use disorders. According to the National Center on Addiction and Substance Abuse [2], the abuse of substances by adolescents affects their physical, mental, academic and interpersonal relationships. Findings from the Office of Applied Studies [3] suggest a lifetime co-occurrence rate of $51.9 \%$ for alcohol use disorders and $34.5 \%$ for substance abuse among men. In the case of women, reported lifetime comorbidity rates of $27.9 \%$ for alcohol use disorder and $26.9 \%$ for substance abuse disorder were reported [4].

Abundant evidence therefore suggest that availability, genetics, personality and social environment all contribute to substance abuse vulnerability among adolescents, with strong evidence revealing the role of stress in the maintenance and sustenance of the phenomenon [5]. Substance abuse occurs most frequently among adolescents

Manuscript submitted March 8, 2013; revised April 30, 2013.

Ikechukwu Uba, Siti Nor Yaacob, Mansor Abu Talib, and Rohani Abdullah are with the Department of Human Development \& Family Studies, Faculty of Human Ecology, UPM, 44300, Serdang, Selangor, Malaysia (e-mail: ubaikechukwu@yahoo.com,_sitinor@putra.upm.edu.my, mansorat@putra.upm.edu.my, rohani.safri@gmail.com).

Sakineh Mofrad is with the Sunway University, Bandar Sunway, Petaling Jaya, 46150, Malaysia (e-mail: sakinehm@sunway.edu.my). with stress disorders [6], and stress disorders were related to frequent alcohol usage [7]. It can also influence illness, either through changing health-related behaviour's such as smoking or via physiological pathways [8]. Studies thus far have found a positive relationship between stress and substance abuse [9], [10] with stress mostly resulting to substance abuse. Although, most literatures reviewed found a significant and positive correlation between stress and substance abuse, however, a few studies found no relationship between the constructs [11]. Studies with no relationship between the concepts literatures revealed were conducted among females, mostly those above 19 years.

Emerging studies on the other hand have found a significant negative relationship between stress and self-esteem among college students [12], [13]. Comparatively, result emanating from a recent study reveals a pattern suggesting the reduction of stress through high self-esteem [14]. Self-esteem is not only the fundamental element of mental health, the scholars argued that it also protects and contributes to improved health and confident social behavior, because it buffers harmful effects [15]. An adolescent's self-esteem is an enormous segment of their self- empathy that is likely to fluctuate and is receptive to both overt and covert influence [16]. Self-esteem is extensively documented as a vital facet of psychological work during adolescence. Stressful life issues were significantly reduced among adolescents with high self-esteem [17]. More so, both earlier and later studies [18], [19] have found significant and negative correlations between self-esteem and substance abuse among adolescents.

Despite the intricate pattern of relationship between substance abuse, stress and self-esteem, no study to our knowledge has attempted to examine the mediating effect of self-esteem in the relationship between stress and substance abuse, regardless of the obvious relationships among the variables. In view of the above short-comings, the present research responded to following research questions.

1) What is the level of stress, self-esteem and substance abuse?

2) What is the relationship between stress, self-esteem and substance abuse?

3) Does self-esteem mediate the relationship between stress and substance abuse?

\section{MATERIALS AND METHODS}

\section{A. Participants}

Given that substance abuse among adolescents starts from 
schools, the sample for the study was derived from schools. The participants of the study comprised of adolescent's between 13 to 18 years $(\mathrm{M}=15.48, \mathrm{SD}=1.53)$ from randomly selected schools in Somolu local government area of Lagos, Nigeria. The specific age bracket was chosen because emerging studies from other parts of the globe highlight the importance of the period in the life course of adolescents. Three hundred and fifty two respondents were eligible for the study. Of the number, $192(54.5 \%)$ were males, while 160 $(45.5 \%)$ were females.

\section{B. Procedure}

Ethical approval for the study was obtained from the Lagos State Ministry of Education. Due to the difficulty associated with the listing of the target population, schools within Somolu Local Government were selected randomly from a list of schools, one school from the North and the other from the South of the local government area. Four research assistants assisted the researcher in the process of data collection. Pertinent issues like confidentiality and the voluntary nature of their participation were read to the respondents after meeting the research sample criteria. Data for the study was collected using the multi-stage cluster sampling technique. In each class sampled, information regarding the study was collected during a two-hour period.

To ensure the proper filling of the scales, the research assistants read the questions aloud, while other volunteer assistants inspected the measure filling process for clarity and understanding by the participant. However, despite the efforts made by the research assistants in ensuring the proper filling of the instruments, 20 instrument booklets were rendered void due to incomplete and in-consistent responses.

\section{Measures}

Each booklet contained a number of validated scales and demographic questions. The respondents also provided details such as their age, gender, number of siblings and other relevant demographic information.

Self-esteem was measured with the Rosenberg Self-Esteem Scale [20]. The 10-item Likert gauge is rated on a 4-point scale ranging from strongly dis-agrees to strongly agree. The scale has been used globally in the assessment of self-esteem. Some example of questions includes: "At times I think I am no good at all" "I feel I do not have much to be proud of" and "I certainly feel useless at times". The scores of the RSS were obtained by reversing the scores on items 1,3 , 4, 7 and 10 and then summing all the scores of the 10 items. The higher the score obtained in the RSS, the greater the level of self-esteem. The RSS has acceptable internal consistency, with a Cronbach alpha coefficient of between .85- .88. In the current study, the Cronbach alpha coefficient obtained was .90

Stress was measured with the Perceived Stress Scale (PSS) [21]. The 14 item Likert instrument is scored on a 5-point scale ranging from never to very often. The scale was used in assessing the degree to which situations in life are appraised stressful. Some example of questions includes: "How often have you been upset because of something that happened unexpectedly"? "How often have you felt that you were unable to control the important things in your life"? "How often have you felt nervous and "stressed". The scores of the PSS were obtained by reversing the scores on items 4, 5, 6, 7, 9,10 and 13 and then summing all the scores of the 14 items. The measure has been widely used in several studies. The higher the score obtained in the PSS, the higher the level of stress. The PSS has acceptable reliabilities ranging from 0.84 to 0.86 . In the present study, the Cronbach alpha coefficient obtained was 0.94 .

Substance abuse was measured with the Drug Abuse Screening Test (DAST) [22]. The 20 items self-report device is scored on a 2-point scale ranging from no to yes, "no" is scored as 0 , and a "yes" is scored as 1 . The scale was used in evaluating the degree to which adolescents abuse substances. Some example of questions includes: "Have you used drugs other than those required for medical reasons"? "Have you abused prescription drugs"? "Do you abuse more than one drug at a time"? The scores of DAST were obtained by reversing the scores on items 4 and 5 and then summing all the score of the 20 items. The measure has been extensively used in numerous studies. The higher the score obtained in DAST, the higher the level of substance abuse. The internal consistency as reported by the authors was between 0.74-0.92. In the present study, the Cronbach alpha coefficient obtained was 0.86 .

\section{Data Analysis}

The data from the research instrument were numerically scored and quantified. Each of the quantitative scores was entered into SPSS version 20 for analysis. Inferential and descriptive statistical tests were also performed. Specifically regression analysis was applied to determine the mediating role of self-esteem in the relationship between stress and abuse. Descriptive statistics were used to calculate means, standard deviation (SD), and range. The interpretation of correlation was based on Cohen's [23] guideline $r=0.10$ to 0.29 small correlation, $r=0.30$ to 0.49 medium correlation and $r=0.50$ to 1.0 large correlation.

\section{RESUlTS AND DisCUSSION}

Table I revealed that 7(2\%) of the adolescents in the study had no substance abuse problems, 67(19\%) were low substance abusers, 53(15.1\%) were moderate substance abusers, $130(36.9 \%)$ were substantial substance abusers, while 95(27\%) were severe substance abusers.

In the case of stress, a substantial number $303(86.1 \%)$ of the adolescents surveyed for the study experienced low stress, while $15(4.3 \%)$ were moderately stressed and $34(9.7 \%)$ were highly stressed. Besides, a significant number $316(89.8 \%)$ of the adolescents surveyed reported high self-esteem, while $28(8.0 \%)$ reported moderate self-esteem and $8(2.3 \%)$ reported low self-esteem.

Furthermore, a multiple regression analysis was conducted to examine the relationship between stress, self-esteem and substance abuse. As outliers can considerably distort the relationship between the predictor and outcome the variables, their influence was examined in the regression equation [24]. In this vein, Cook's distance measure was used to examine the influence possible outliners may have on the model. However, after the examination, Cook's distance measure 
did not identify any problem with the model [25].

\begin{tabular}{|c|c|c|c|c|}
\hline Variables score & Level & $\mathrm{n}(\%)$ & Mean & SD \\
\hline Substance Abuse & & & 11.00 & 5.14 \\
\hline 0 & No Problem & $7(2.0)$ & & \\
\hline $1-5$ & $\begin{array}{l}\text { Low Level } \\
\text { Problem }\end{array}$ & $67(19.0)$ & & \\
\hline $6-10$ & $\begin{array}{l}\text { Moderate } \\
\text { Level Problem }\end{array}$ & $53(15.1)$ & & \\
\hline $11-15$ & $\begin{array}{l}\text { Substantial } \\
\text { Level Problem }\end{array}$ & 130(36.9) & & \\
\hline $16-20$ & $\begin{array}{l}\text { Severe Level } \\
\text { Problem }\end{array}$ & $95(27.0)$ & & \\
\hline Stress & & & 30.24 & 12.51 \\
\hline$<=46.00$ & Low & $303(86.1)$ & & \\
\hline $47.00-48.00$ & Moderate & $15(4.3)$ & & \\
\hline $49.00+$ & High & $34(9.7)$ & & \\
\hline Self-Esteem & & & 24.76 & 7.53 \\
\hline$<=14.00$ & Low & $8(2.3)$ & & \\
\hline $15.00-15.00$ & Moderate & $28(8.0)$ & & \\
\hline $16.00+$ & High & $316(89.8)$ & & \\
\hline
\end{tabular}

In consonance with Baron and Kenny [26], three conditions must exist for mediation to occur. First, the independent variable (stress) must be significantly related with the dependent variable (substance abuse), in the absence of the mediator (self-esteem). Second, the independent variable (stress) must be significantly related to the mediator (self-esteem). Finally, the mediator (self-esteem) must be significantly related to the dependent variable (substance abuse). Furthermore, the addition of the mediator (self-esteem) into the regression model must reduce (partial mediation) or eliminate (full mediation) the initial effect of the independent variable (stress) on the dependent variable (substance abuse).

In the present study, zero-order correlations (see Table II) indicated that the first three criteria for mediation had been met. Table 2 indicated a direct effect of stress on substance abuse $(B=.148, \mathrm{SE}=.020, t=7.223, p<.05)$ and self-esteem $(B=-.324, \mathrm{SE}=.027, t=-11.947, p<.05)$. The relationship between self-esteem (mediator) and substance abuse (dependent variable) was also significant $(B=-.366, \mathrm{SE}=$. $031, t=-11.868, p<.05$ ). In the fourth regression step (Table 2 ), after controlling the effect of stress, the result validates the notion of complete mediation. This is because the relationship between stress and substance abuse was approaching zero and therefore non-significant $(B=.042, \mathrm{SE}$ $=.022, p>.05)$.

The extent of mediation was found by subtracting the unstandardized beta coefficient $(B)$ in the fourth step of the multiple regression $(B=.042)$ when stress was controlled for (table 2) from the first step $(B=.148)$ (Direct effect of stress on substance abuse). The result therefore was .148 - .042 $=.106$. As recommended by Baron and Kenny [26], the mediation effect was tested using Sobel test. In conducting Sobel test, the unstandardized coefficients and standard error of path $\boldsymbol{a}$, stress and self-esteem $(B=-.324, \mathrm{SE}=.027)$ and path $\boldsymbol{b}$, self-esteem and substance abuse $(B=-.366, \mathrm{SE}=.31)$ were assessed using Sobel test [29] [30]. The z-value for the indirect path was $8.42, p<.05$, indicating the significant mediation effect of self-esteem in the relationship between stress and substance abuse.
TABLE II: MultiPle REgRESSION OF STRESS AND SubSTANCE ABUSE MEDIATED BY SELF-ESTEEM $(N=352)$

\begin{tabular}{lllllll}
\hline Step & IV & DV & B & SE & Beta & t \\
\hline $\mathbf{1}$ & Stress & $\begin{array}{l}\text { Substance } \\
\text { abuse }\end{array}$ & $.148^{* *}$ & .020 & .360 & 7.223 \\
$\mathbf{2}$ & Stress & $\begin{array}{l}\text { Self-esteem } \\
\mathbf{3}\end{array}$ & $-.324^{* *}$ & .027 & -.538 & -11.947 \\
& Self-esteem & $\begin{array}{l}\text { Substance } \\
\text { abuse }\end{array}$ & $-.366^{* *}$ & .031 & -.536 & -11.868 \\
\hline $\mathbf{4}$ & Stress & $\begin{array}{l}\text { Substance } \\
\text { abuse }\end{array}$ & .042 & .022 & .101 & 1.896 \\
& Self-esteem & & $-.328^{* *}$ & .036 & -.481 & -9.019 \\
\hline
\end{tabular}

Note: $B=$ Unstandardized coefficient; Beta $=$ Standardized coefficient $*$ $p<.05, * * p<.01$

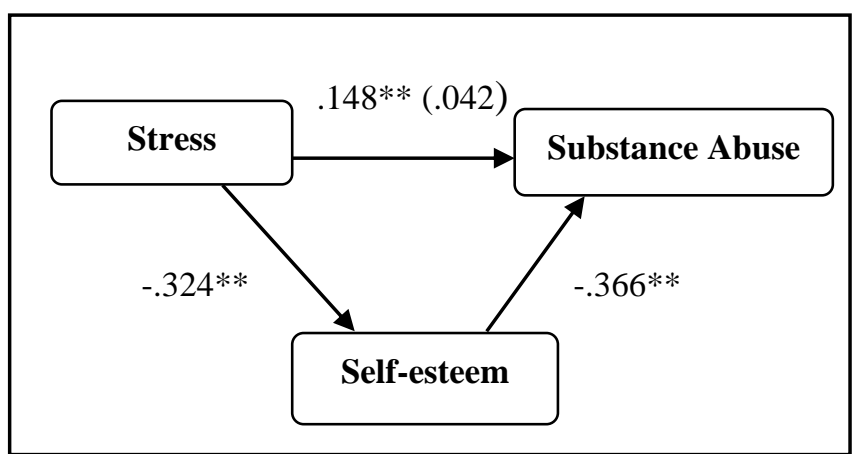

Fig. 1. The mediator role of self-esteem in the relationship between stress and substance abuse.

The finding is in line with studies [19], [27] that revealed the effect of self-esteem in the diminution of substance abuse. Self-esteem is the most consistent predictor of the likelihood and extent of substance abuse [19].

In line with the above studies, Bandura [28] argued that noticeable self-esteem influences every stage of change in substance abuse, the initiation of changes, their achievement, recuperation from relapse, and long-term continuation of abstinence. This indicated that adolescents with high self-esteem succeeded more in their effort to abstain from substance abuse. To affirm the findings of the study, a recent study [19] revealed adolescents with high self-esteem as less likely to have smoked cigarettes, drank alcohol, or used marijuana, and among those adolescents used alcohol and marijuana, those with high self-esteem were more likely to have limited their extent of usage. The study also suggested that besides the prediction of substance abuse by stress, adolescents with high self-esteem can overcome the effect of stress. Essentially, people are shaped by the interactions between their behaviors, thoughts, and environmental events [28].

\section{CONCLUSION}

The current study investigated the mediation effect of self-esteem in the relationship between stress and substance abuse among adolescents. The findings suggested that adolescents with high self-esteem can overcome the effect of stress and were less likely to abuse substances. Based on cognitive theory [28], adolescents are shaped by the interactions between their behaviors, thoughts, and environmental events, meaning that adolescents with higher self-esteem can overcome the challenges of their environment. The study therefore confirmed the role of self-esteem as a mediator in the relationship between stress 
and substance abuse. Recommendations of the study highlighted the need for greater enlightenment on the importance of self-esteem particularly among adolescents.

\section{REFERENCES}

[1] L. Schwabe, A. Dickinson, and O.T. Wolf, "Stress, habits, and drug addiction: A psycho-neuroendocrinological perspective," Experimental and Clinical Psychopharmacology., vol. 19, no. 1, pp. 53-63, 2011.

[2] National Center on Addiction and Substance Abuse. Adolescent substance use: America's public health problem. [Online] Available: http://www.eric.ed.gov/PDFS/ED521379.pdf

[3] Office of Applied Studies. Results from the 2004 National Survey on Drug Use and Health: National findings (DHHS Publication No. SMA 05-4062, NSDUH Series H-28). Rockville, MD: Substance Abuse and Mental Health Services Administration. [Online] Available: http://www.oas.samhsa.gov/nsduh/reports.htm 2005.

[4] M. E. D. Murphy, G. R. Parra, M. T. Shea, S. Yen, C. M. Grilo, C. A. Sanislow, and J. C. Markowitz, "Trajectories of PTSD and substance use disorders in a longitudinal study of personality disorders," Psychological Trauma: Theory, Research, Practice, and Policy, vol. 1 , no. 4, pp. 269-281, 2009.

[5] R. Sinha, "Chronic Stress, Drug Use, and Vulnerability to Addiction," Annals of the New York Academy of Sciences, vol. 1141, pp. 105-130, 2008.

[6] M. W. Miller, D. S. Vogt, S. L. Mozley, D. G. Kaloupek, and T. M. Keane, "PTSD and substance-related problems: The mediating roles of disconstraint and negative emotionality," Journal of Abnormal Psychology, vol. 115, no. 2, pp. 369-379, 2006.

[7] R. E. Adams, J. A. Boscarino, and S. Galea, "Alcohol use, mental health status, and psychological well-being 2 years after the World Trade Center attacks in New York City," Journal of Drug and Alcohol Abuse, vol. 32, pp. 203-204, 2006.

[8] Health Psychology, Learning objectives. (2011). [Online] Available: http://www.blackwellpublishing.com/intropsych/pdf/chapter19.pdf

[9] S. H. Stewart, P. J. Conrod, R. O. Pihl, and M. Doniger, "Relationships between posttraumatic stress symptom dimensions and substance dependence in a community-recruited sample of substance-abusing women," Psychology of Addictive Behaviors, vol. 13, pp. 78-88, 1999.

[10] C. M. Chemtob, Y. Nomura, L. Josephson, R. E. Adams, and L. Sederer, "Substance use and functional impairment among adolescents directly exposed to the 2001 World Trade Center attacks," Disasters, vol. 33, pp. 337-352, 2009.

[11] A. Ro and K. H. Choi, "Effects of gender discrimination and reported stress on drug use among racially/ethnically diverse women in Northern California," Women's Health Issues, vol. 20, no. 3, pp. 211-218, 2010.

[12] S. Dixon and S. E. R. Kurpius, "Depression and college stress among university undergraduates: Do mattering and self-esteem make a difference?" Journal of College Student Development, vol. 49, pp. 412-424, 2008.

[13] D. Edwards, P. Burnard, K. Bennett, and U. Hebden, "A longitudinal study of stress and self-esteem in student nurses," Nurse Education Today, vol. 30, pp. 78-84, 2010.

[14] F. Griva and F. Anagnostopoulus, "Positive psychological states and anxiety: the mediating effect of proactive coping," Psychology Rep. vol. 107, no. 3, pp. 795-804, 2010.

[15] M. Mann, C. M. Hosman, H. P. Schaalma, and N. K D. Vries, "Self-esteem in a broad-spectrum approach for mental health promotion," Health Education Research, vol. 19, pp. 357-372, 2004.
[16] J. R. Z. Abela, C. A. Webb, C. Wagner, M. R. Ho, and P. Adams, “The role of self-criticism, dependency, and hassles in the course of depressive illness: A multiwave longitudinal study," Personality and Social Psychology Bulletin, vol. 32, pp. 328-338, 2006.

[17] U. Orth, R. Robins, and L. L. Meier, "Disentangling the effects of low self-esteem and stressful life events on depression: Findings from three longitudinal studies," Journal of Personality and Social Psychology, vol. 97, pp. 307-321, 2009 .

[18] J. H. Liem, E. C. Cavell, and K. Lustig, "The influence of authoritative parenting during adolescence on depressive symptoms in young adulthood: Examining the mediating roles of self-development and peer support," The Journal of Genetic Psychology, vol. 171, no. 1, pp. 73-92, 2010.

[19] B. L. Zamboanga, S. J. Schwartz, L. H. Jarvis, and K. V. Tyne, "Acculturation and substance use among Hispanic early adolescents: Investigating the mediating roles of acculturative stress and self-esteem," Journal of Primary Prevention, vol. 30, pp. 315-333, 2009

[20] M. Rosenberg, Society and the Adolescent Self-Image, Princeton, NJ: Princeton University Press, 1965.

[21] S. Cohen, T. Kamarck, and R. Mermelstein, "A Global Measure of Perceived Stress," Journal of Health and Social Behavior, vol. 24, pp. 385-396, 1983.

[22] H. A. Skinner, "Drug abuse screening test," Addictive Behaviour, vol. 7, pp. 363-371, 1982.

[23] J. Cohen, Statistical Power Analysis for Behavioural Sciences, Hillsdale, NJ: Erlbaum, 1988.

[24] J. Neter, W. Wasserman, and M. H. Kutner, Applied Linear Regression Models, 2nd Edition. Irwin Homewood IL, 1989.

[25] R. D. Cook and S. Weisberg, "Criticism and influence in regression," in Sociological Methodology, S. Leinhardt, ed., San Francisco: Jossey-Bass Publishers, pp. 313-362, 1982.

[26] R. M. Baron and D. A. Kenny, "The Moderator-Mediator Variable Distinction in Social Psychological Research: Conceptual, Strategic, and Statistical Considerations," Journal of Personality and Social Psychology, vol. 51, no. 6, pp. 1173-1182, 1986.

[27] R. C. Swaim and J. C. Wayman, "Multidimensional self-esteem and alcohol use among Mexican American and White Non-Latino adolescents: Concurrent and prospective effects," American Journal of Orthopsychiatry, vol. 74, pp. 559-570, 2004.

[28] A. Bandura, Self-Efficacy: The Exercise of Control, New York: W.H Freeman, 1997.

[29] M. E. Sobel, "Asymptotic confidence intervals for indirect effects in structural equation models," in Sociological Methodology, S. Leinhardt (Ed.), Washington DC: American Sociological Association, pp. 290-312, 1982

[30] D. S. Soper. (2013). Sobel Test Calculator for the Significance of Mediation. [Online] Available: http://www.danielsoper.com/statcalc

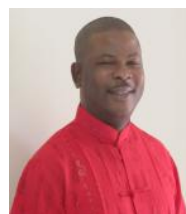

Ikechukwu Uba is a developmental psychologist from University Putra Malaysia. He is majored in Human Development. His research interest revolves around human developmental issues across the life span. He has authored and co-authored many academic papers, books and book chapters. 